\title{
Utilisation rationnelle de fertilisants organiques pour la production de macroinvertébrés benthiques d'eau douce en pisciculture
}

\author{
Richard Adande (1) M.N. Dine Liady ${ }^{(1)}$, H.K. Janvier Bokossa ${ }^{(1)}$, Gildas Djidohokpin ${ }^{(1)}$, \\ Fouad Zouhir $^{(2)}$, G. Apollinaire Mensah ${ }^{(3)}$, E. Didier Fiogbe ${ }^{(1)}$ \\ (1) Université d'Abomey-Calavi (UAC). Faculté des Sciences et Techniques. Département de Zoologie. Laboratoire de \\ Recherche sur les Zones Humides (LRZH). BP 526. Cotonou (Bénin). E-mail : richard_adande@yahoo.fr \\ (2) Université de Liège. Département Sciences et Gestion de l'Environnement. Unité Assainissement et Environnement. \\ Avenue de Longwy, 185. BE-6700 Arlon (Belgique). \\ ${ }^{(3)}$ Institut National de Recherches Agronomiques du Bénin (INRAB). 01 BP 2359. Cotonou (Bénin).
}

Reçu le 30 mai 2017, accepté le 6 septembre 2018, mis en ligne le 8 octobre 2018.

Cet article est distribué suivant les termes et les conditions de la licence CC-BY (http://creativecommons.org/licenses/by/4.0/ deed.fr)

Description du sujet. Cette étude détermine les conditions optimales d'utilisation de déjections de lapin dans la production de macroinvertébrés benthiques pour une production de poisson à faible cout.

Objectifs. L'objectif de cette étude est la détermination de la dose optimale de déjections pour une production plurispécifique de macroinvertébrés d'eau douce.

Méthode. Pour ce faire, six traitements dont un témoin ont reçu respectivement $0 \%, 10 \%, 25 \%, 50 \%, 75 \%$ et $100 \%$ de déjections par rapport au volume total de substrat. Les cultures ont été réalisées dans des seaux qui ont chacun reçu au total $10 \mathrm{dm}^{3}$ de substrat, $16 \mathrm{dm}^{3}$ d'eau de forage et $4 \mathrm{dm}^{3}$ d'eau d'étang. La densité initiale d'ensemencement est de 6 ind $\cdot \mathrm{dm}^{-2}$ d'Odonates et d'Annélides (soit 3 ind $\cdot \mathrm{dm}^{-3}$, pour une profondeur de $0,5 \mathrm{~m}$ ), 8 ind $\cdot \mathrm{dm}^{-2}$ de Chironomidae (soit 4 ind $\cdot \mathrm{dm}^{-3}$ ) et 10 ind $\cdot \mathrm{dm}^{-2}$ de Mollusques (soit $5 \mathrm{ind} \cdot \mathrm{dm}^{-3}$ ). Les paramètres physico-chimiques et biologiques de la culture ont été suivis tout au long des 63 jours d'expérimentation.

Résultats. Les densités les plus élevées de macroinvertébrés benthiques sont obtenues avec le traitement $\mathrm{T}_{2}(192 \pm 1,20$ ind. $\mathrm{dm}^{-2}$ de Chironomidae, $52 \pm 0,8$ ind $\cdot \mathrm{dm}^{-2}$ de Mollusques, $6 \pm 0,33$ ind $\cdot \mathrm{dm}^{-2} \mathrm{~d}$ 'Odonates et $4 \pm 0,33$ ind $\cdot \mathrm{dm}^{-2} \mathrm{~d}$ 'Annélides soit, $96 \pm 1,20$ ind $\cdot \mathrm{dm}^{-3}$ de Chironomidae, $26 \pm 0,8 \mathrm{ind} \cdot \mathrm{dm}^{-3} \mathrm{de}$ Mollusques, $3 \pm 0,33 \mathrm{ind} \cdot \mathrm{dm}^{-3} \mathrm{~d}$ 'Odonates et $2 \pm 0,33 \mathrm{ind} \cdot \mathrm{dm}^{-3}$ d'Annélides). La biomasse la plus élevée des Chironomidae et des Mollusques est obtenue avec $\mathrm{T}_{2}$, soit respectivement $728,1 \pm$ $2,23 \mathrm{mg}$ de matière sèche $\cdot \mathrm{dm}^{-2}$ et $699,98 \pm 22,49 \mathrm{mg}$ de matière sèche $\cdot \mathrm{dm}^{-2}$ (ou encore respectivement $364,05 \pm 2,23 \mathrm{mg}$ de matière sèche $\cdot \mathrm{dm}^{-3}$ et $349,99 \pm 22,49 \mathrm{mg}$ de matière sèche $\left.\cdot \mathrm{dm}^{-3}\right)$.

Conclusions. Au regard de ces résultats, le traitement $\mathrm{T}_{2}\left(140 \mathrm{~g}\right.$ de matière sèche $\cdot \mathrm{dm}^{-2}$, soit $75 \mathrm{~g}$ de déjections de lapin sèches. $\mathrm{dm}^{-3}$ de substrat) constituerait la dose à recommander pour une production plurispécifique optimale de macroinvertébrés benthiques d'eau douce.

Mots-clés. Pisciculture, déjection, lapin, production, macroinvertébrés.

Rational utilization of organic fertilizers for freshwater benthic macroinvertebrate production in fish farming Description of the subject. This study aims to determine optimal conditions of rabbit manure utilization in freshwater benthic macroinvertebrate production in order to produce fish at low cost.

Objectives. To determine the optimal dose of rabbit manure to be used for freshwater macroinvertebrate production.

Method. Six treatments, including one control, received respectively 0\%, 10\%, 25\%, 50\%, 75\% and 100\% of rabbit manure compared to the substrate total volume. Cultures were carried out in buckets, which received $10 \mathrm{dm}^{3}$ of substrate, $16 \mathrm{dm}^{3}$ of groundwater and $4 \mathrm{dm}^{3}$ of fishpond water. The initial seeding density was 6 ind $\cdot \mathrm{dm}^{-2}$ of odonates, $6 \mathrm{ind} \cdot \mathrm{dm}^{-2}$ of annelids or (3 ind $\cdot \mathrm{dm}^{-3}$, for $\left.0.5 \mathrm{~m} \mathrm{depth}\right), 8 \mathrm{ind} \cdot \mathrm{dm}^{-2}$ of chironomids or $\left(4 \mathrm{ind} \cdot \mathrm{dm}^{-3}\right)$ and 10 ind $\cdot \mathrm{dm}^{-2}$ of mollusks or $\left(5 \mathrm{ind} \cdot \mathrm{dm}^{-3}\right)$. Physicochemical and biological parameters were measured during the 63 days of the experiment. 
Results. The highest densities of benthic macroinvertebrates were obtained within treatment $\mathrm{T}_{2}$, with $192 \pm 1.20 \mathrm{ind} \cdot \mathrm{dm}^{-2}$ of chironomids, $52 \pm 0.8 \mathrm{ind} \cdot \mathrm{dm}^{-2}$ of mollusks, $6 \pm 0.33 \mathrm{ind} \cdot \mathrm{dm}^{-2}$ of odonates and $6 \pm 0.33 \mathrm{ind} \cdot \mathrm{dm}^{-2}$ of annelids. Considering the depth of our experimental buckets $(0.5 \mathrm{~m})$, these highest densities also corresponded to respectively: $96 \pm 1.20 \mathrm{ind} \cdot \mathrm{dm}^{-3}$ of chironomids, $26 \pm 0.8 \mathrm{ind} \cdot \mathrm{dm}^{-3}$ of mollusks, $3 \pm 0.33 \mathrm{ind} \cdot \mathrm{dm}^{-3}$ of odonates and $2 \pm 0.33 \mathrm{ind} \cdot \mathrm{dm}^{-3}$ of annelids). The highest biomass of chironomids and mollusks was obtained within treatment $T_{2}$, with respectively $728.1 \pm 2.23 \mathrm{mg}$ dry matter $\cdot \mathrm{dm}^{-2}$ and $699.98 \pm 22.49 \mathrm{mg}$ dry matter $\cdot \mathrm{dm}^{-2}$ (i.e. respectively $364.05 \pm 2.23 \mathrm{mg}$ dry matter $\cdot \mathrm{dm}^{-3}$ and $349.99 \pm 22.49 \mathrm{mg}$ dry matter $\left.\cdot \mathrm{dm}^{-3}\right)$.

Conclusions. Considering these results, treatment $\mathrm{T}_{2}$ with $140 \mathrm{~g}$ dry matter of rabbit manure $\cdot \mathrm{dm}^{-2}$ of substrate (i.e. $75 \mathrm{~g}$ dry matter of rabbit manure $\mathrm{dm}^{-3}$ of substrate) could constitute the optimal dose that could be recommended for the optimal production of freshwater benthic macroinvertebrates.

Keywords. Fish farming, rabbit manure, production, macroinvertebrates.

\section{INTRODUCTION}

Face au ralentissement des prises halieutiques, aussi bien d'eau douce que d'eau saumâtre ou marine, la croissance des productions aquacoles est considérée comme une voie de réponse à l'augmentation de la demande générée par la croissance de la population mondiale qui pourrait atteindre 9,6 milliards d'habitants d'ici 2050 (UNDESA, 2013 ; Rey-Valette, 2014). Par exemple, le déficit en produits halieutiques à combler par le Bénin en 2025 est estimé à 34040 t (Rurangwa et al., 2014). En réponse à la croissance des populations et à celle de leurs besoins alimentaires, l'aquaculture peut jouer un rôle en aidant à augmenter la production piscicole à partir de l'utilisation de fertilisants organiques (Schlumberger \& Bouretz, 2002). Les fertilisants organiques sont capables d'augmenter considérablement, et à moindre cout, la production d'aliments naturels dans les étangs et, par ricochet, la croissance et le rendement des poissons pour une production aquacole plus écologique et durable (Agadjihouèdé et al., 2011; Sorgeloos, 2014). Les proies vivantes constituent une source de nourriture primordiale à la production des alevins de poissons-chats africains (Agadjihouèdé et al., 2011). Parmi les proies vivantes, les macroinvertébrés benthiques constituent une composante importante des écosystèmes aquatiques de par leur activité de bioturbation à l'interface eau-sédiment qui facilite la disponibilité des nutriments (Risnoveanu et al., 2004 ; Biswas et al., 2009) ainsi que leur intervention dans la transformation de la matière organique des écosystèmes (Hoómann, 2005 ; Waite et al., 2010). De même, ils assurent les transferts d'énergie entre la matière organique et les maillons supérieurs de la chaine trophique et sont d'excellents bioindicateurs (Ramesh \& Jitendra, 2009 ; Uherek \& Gouveia, 2014 ; Fierro et al., 2017). Ils sont la source principale de nourriture pour plusieurs espèces de poissons (e.g. Clarias gariepinus, Mallotus villosus; Kuhlmann et al., 2001 ; Akodogbo et al., 2016). Leur utilisation est indispensable à la réussite de l'élevage postlarvaire et juvénile (López van Oosterom et al., 2013).
En effet, selon Serra et al. (2015), les Chironomidae (vers de sang, Diptère) représentent $50 \%$ des insectes benthiques des eaux douces et constituent, avec les vers (oligochètes), une source de nourriture appréciée par les poissons (Evangelista et al., 2005 ; Serra et al., 2016). Selon Sulistiyarto \& Yulintine (2014), les poissons au stade post-larvaire nourris avec des larves de Chironomidae ont donné des taux de survie et de croissance plus élevés que ceux soumis aux aliments artificiels. Ainsi, les Chironomidae constituent une source d'alimentation de qualité pour une bonne croissance des juvéniles de poissons (Bogut et al., 2007 ; Cartier et al., 2011 ; Hussain \& Pandit, 2012). Ces macroinvertébrés sont riches en protéines, lipides, vitamines et en sels minéraux tels que le fer (Bogut et al., 2007 ; Maleknejad et al., 2014).

Malgré le rôle des macroinvertébrés benthiques dans la nutrition des poissons, très peu de données sur leur production à partir de fertilisants organiques d'origine animale, comme les déjections de lapin, sont disponibles dans la littérature. Cette étude a donc pour objectifs :

- d'évaluer l'influence d'un fertilisant organique sur l'abondance de quatre taxons de macroinvertébrés benthiques,

- d'évaluer la biomasse de Chironomidae et de Mollusques,

- de déterminer la dose optimale de déjections de lapin pour une production optimale de macroinvertébrés.

\section{MATÉRIEL ET MÉTHODES}

\subsection{Dispositif expérimental}

Le dispositif expérimental (Figure 1) est constitué de 18 seaux en plastique de 801 de capacité, exposés à l'air libre sur le site du Laboratoire de Recherche sur les Zones Humides (LRZH), Station de Recherche sur la diversification de la pisciculture de l'Université d'Abomey-Calavi (UAC) au Bénin.

Ce dispositif a été utilisé pour étudier six traitements dont un témoin sans déjections de lapin $\left(\mathrm{T}_{0}, \mathrm{~T}_{1}, \mathrm{~T}_{2}, \mathrm{~T}_{3}\right.$, 


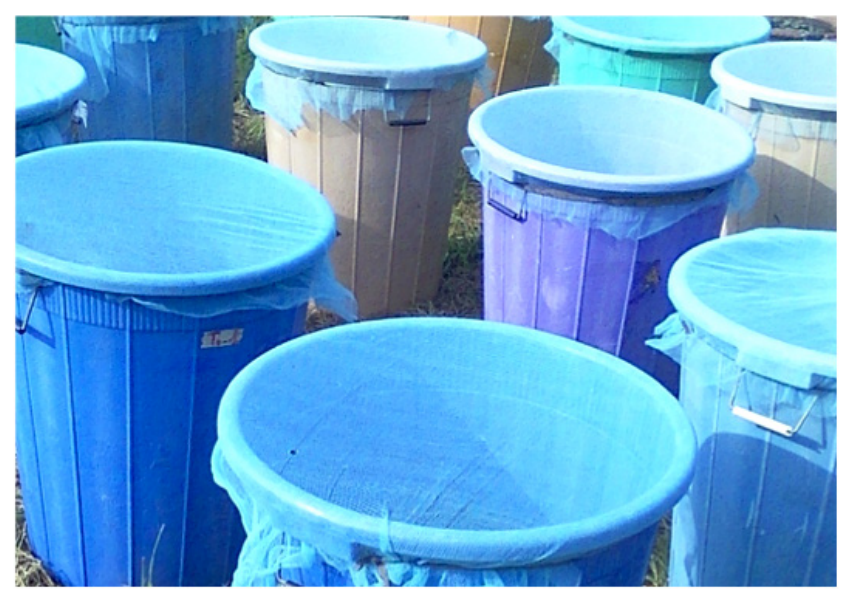

Figure 1. Dispositif expérimental de production de macroinvertébrés benthiques d'eau douce - Experimental set-up for freshwater benthic macroinvertebrates production.

$\mathrm{T}_{4}$, et $\mathrm{T}_{5}$ ) réalisés en triplicat (Figure $\mathbf{1}$ ). À cet effet, $72 \mathrm{~h}$ avant le début de l'expérimentation, les seaux ont été nettoyés et désinfectés. Chaque seau a reçu $10 \mathrm{dm}^{3}$ de substrat, $16 \mathrm{dm}^{3}$ d'eau de forage et $4 \mathrm{dm}^{3}$ d'eau d'étang préalablement filtrée à l'aide d'un tamis métallique de $200 \mu \mathrm{m}$ vide de maille, afin d'éliminer d'éventuels macroinvertébrés (Akodogbo et al., 2016). Le substrat utilisé est constitué d'un mélange de sable, de graviers et de déjections de lapin préalablement moulues. Ces déjections sont issues de lapins soumis à un régime alimentaire à faible cout, assurant de bonnes performances sur leur production (Adande et al., 2017). Celui-ci est constitué de $2 \%$ de cossette de manioc, $30 \%$ de son de maïs, $10 \%$ de tourteau de palmiste, $10 \%$ de tourteau de soja, $5 \%$ de tourteau de coton, $2 \%$ de coquille, $10 \%$ de drêche, $5 \%$ de levure de bière, $15 \%$ d'Azolla filiculö̈des, $10 \%$ de Panicum maximum et $1 \%$ de sel. Ces déjections de lapin sont constituées de $15 \%, 1,3 \%$ et $0,8 \%$ respectivement de $\mathrm{N}, \mathrm{P}$ et $\mathrm{K}$.

Au démarrage de l'expérience, les traitements $\mathrm{T}_{0}$, $\mathrm{T}_{1}, \mathrm{~T}_{2}, \mathrm{~T}_{3}, \mathrm{~T}_{4}$, et $\mathrm{T}_{5}$ ont été fertilisés respectivement avec $0 \%, 10 \%, 25 \%, 50 \%, 75 \%$ et $100 \%$ de déjections de lapin par rapport au volume total du substrat, conformément à Akodogbo et al. (2016). Trois jours après cette fertilisation, les seaux ont été ensemencés de macroinvertébrés à un nombre initial de 30 individus composés de 6 Odonates, 6 Annélides, 8 Chironomidae et 10 Mollusques. Les seaux ont été couverts de toile de moustiquaire pour empêcher l'entrée d'éventuels prédateurs de macroinvertébrés dans le milieu de culture. L'échantillonnage a été fait tous les 7 jours à l'aide d'un tamis de $240 \mu \mathrm{m}$ vide de maille. La durée de l'expérience est de 63 jours (9 semaines). Les échantillons ont été conservés dans du formol $(10 \%)$ pour éviter une perte de poids par lixiviation (Leuven et al., 1985 ; Benke et al., 1999). L'identification et le dénombrement des invertébrés benthiques ont été réalisés sous une loupe binoculaire (Leica S8AP0). Les paramètres physico-chimiques (température, $\mathrm{pH}$, conductivité, salinité [due aux déjections de lapin], TDS, oxygène dissous) ont été mesurés in situ tous les 7 jours (à $8 \mathrm{~h}$ et $17 \mathrm{~h}$ ) avec la sonde multi-paramètre CALYPSO (softer version/2015 2138 SN-ODEON CALYPSO ; $\pm 0,1 \mathrm{C}$ sensibilité). Les mesures de turbidité ont été réalisées dans les différents milieux de production à l'aide d'un turbidimètre (EUTECH TN-100). Cinq cents ml d'eau du milieu de production ont été prélevés tous les 7 jours puis conservés au réfrigérateur (à $4{ }^{\circ} \mathrm{C}$ ) pour le dosage des nitrates, nitrites, orthophosphates et l'ammonium au spectrophotomètre d'absorption moléculaire (HACH DR/2800) suivant Rodier \& Merlet (2012). Le volume total de chacun des seaux était réajusté tous les trois jours avec de l'eau distillée pour éviter tout phénomène de concentration.

\section{2. Établissement des relations taille-poids}

Plusieurs auteurs ont mis en cause l'utilisation abusive des relations taille-poids disponibles dans la littérature du fait que, pour une même espèce, celle-ci varie beaucoup en fonction des conditions du milieu, du substrat, des paramètres physico-chimiques, des conditions trophiques et même de la génétique (Benke et al., 1999 ; Miserendino, 2001). Pour ces raisons, des droites de régression tailles-poids ont été préalablement calibrées, sur les taxons qui ont été utilisés dans ce travail, pour permettre d'estimer les biomasses des macroinvertébrés benthiques produites. Pour chacun de ces taxons, une dizaine de classes de taille ont été prises en compte et les données des mesures de poids sec (PS) et de longueur (L) ont été ajustées par la méthode des moindres carrés au modèle exponentiel :

$$
P S=a L^{b}
$$

avec $P S$ : poids sec (mg), $L$ : longueur (mm), $a$ et $b$ : constantes.

Deux spécimens de Chironomidae (ordre des Diptères, sous-ordre des Nématocères) et de Mollusques (classe des Gastéropodes, Melanoides tuberculata) régulièrement cultivés dans ces mêmes conditions depuis quelques mois, ont été choisis à partir de leurs abondances observées durant la période de production. La mesure des longueurs totales (en $\mathrm{mm}$ ) a été faite à l'aide d'un ichtyomètre. Pour déterminer les poids secs des organismes benthiques, des nacelles ont d'abord été séchées pendant $2 \mathrm{~h}$ dans une étuve à $105^{\circ} \mathrm{C}$ puis acclimatées pendant $1 \mathrm{~h}$ dans un dessiccateur, à la température du laboratoire, avant de déterminer par 
pesée leur poids à vide. Ces nacelles ont ensuite été remplies des individus (préalablement rincés trois fois avec de l'eau distillée pour les débarrasser d'éventuels déchets et de la solution de conservation) de chaque classe de taille en triplicat. Les individus ont été séchés à l'étuve à $60^{\circ} \mathrm{C}$ pendant $24 \mathrm{~h}$ (Benke et al., 1999), suivi d'une acclimatation pendant $1 \mathrm{~h}$ au dessiccateur avant d'être pesés à la balance (Ohauscorp.pine Book NJUSA de sensibilité $0,1 \mathrm{mg}$ ). Le poids moyen d'un individu dans chaque classe a été ensuite calculé.

\subsection{Comparaison des performances des différents traitements}

Les performances des six traitements ont été comparées suivant deux méthodes.

La première a consisté en une comparaison des biomasses obtenues dans les différents traitements ; la deuxième a consisté à comparer les taux de croissance spécifiques des organismes durant leurs phases de croissance exponentielle conformément à Liady et al. (2015). En effet, les courbes de croissance en milieu non renouvelé de macroinvertébrés présentant des phases de croissance identiques à celles observées sur des cultures de micro-organismes en milieu non renouvelé, des lois mathématiques identiques ont été considérées. Ainsi, sur chaque traitement, la phase de croissance exponentielle de la biomasse a été considérée et décrite par la relation $\mathrm{P}_{\mathrm{t}}=\mathrm{P}_{\mathrm{o}} \mathrm{e}^{\mu \mathrm{t}}$ (Waldbauer, 1968). À partir de cette relation, le taux de croissance spécifique $(\mu)$ a été déterminé en suivant l'évolution du logarithme de la biomasse relative $\ln$ $\left(\mathrm{P}_{\mathrm{t}} / \mathrm{P}_{\mathrm{o}}\right)$ en fonction du temps; il correspond à la pente de la droite ainsi obtenue.

\subsection{Analyse statistique}

Toutes les données ont été analysées à l'aide du logiciel statistique STATISTICA (Statsoft.inc.). Pour chacun des tests, le seuil de significativité a été fixé à $\alpha=5 \%$.

Les régressions ont été analysées en suivant respectivement la qualité de l'ajustement des données observées au modèle théorique testé, puis la significativité de chaque coefficient, par rapport à 0 . L'influence des traitements a été étudiée à l'aide d'une analyse de variance (ANOVA) à un facteur (dose de déjections) et six modalités. Le cas échéant, la significativité des différences entre les moyennes a été testée à l'aide du test de la plus petite différence significative (LSD de Fisher).

L'intensité des liaisons entre variables numériques (corrélation) a été évaluée à l'aide du test de corrélation de Pearson (test paramétrique) lorsque les résidus présentent une distribution normale; dans le cas contraire, un test de corrélation de Spearman a été appliqué.

\section{RÉSULTATS}

\subsection{Influence des doses sur la qualité de l'eau}

Les résultats de l'analyse de variance de l'effet des différentes doses (traitements), en considérant les jours d'application, indiquent une différence significative $(p<0,00)$ des différentes doses sur les paramètres physico-chimiques (Tableau 1). Les valeurs moyennes des paramètres physico-chimiques enregistrés au cours de cette étude sont présentées dans le tableau 2.

La température des différents traitements a très peu varié. Elle fluctue entre 0,46 et 0,59 autour de $31^{\circ} \mathrm{C}$. En revanche, le $\mathrm{pH}$ varie entre 6,74 et 7,51 avec une différence très hautement significative $\left(\mathrm{F}_{(5,12)}=35,21\right.$; $p<0,00$ ) (Tableaux 1 et 2). En effet, un $\mathrm{pH}$ légèrement acide est obtenu dans $\mathrm{T}_{4}$ et $\mathrm{T}_{5}$, contrairement aux autres traitements qui sont proches de la neutralité. La turbidité la plus élevée est enregistrée dans le traitement $\mathrm{T}_{5}$ avec une différence très hautement significative entre les traitements $\left(\mathrm{F}_{(5,12)}=171,05 ; p<0,00\right)$. De même, les valeurs de conductivité ont fortement varié et augmenté avec la dose de déjections de lapin $\left(F_{(5,12)}=100,59 ; p<0,00\right)$. Elle est presque constante dans les traitements $\mathrm{T}_{0}$ et $\mathrm{T}_{1}$. La richesse en nutriments $\left(\mathrm{N}-\mathrm{NO}_{2}{ }^{-}, \mathrm{N}_{-} \mathrm{NO}_{3}^{-}, \mathrm{N}-\mathrm{NH}_{3}, \mathrm{P}_{-} \mathrm{PO}_{4}^{3-}\right)$ est observée à partir du traitement $\mathrm{T}_{2}$ et la plus forte valeur est obtenue avec le traitement $\mathrm{T}_{5}$ (Tableau 2). L'oxygène varie aussi entre $2,70 \pm 0,40$ et $4,11 \pm 0,1 \mathrm{mg} \cdot 1^{-1}$ et diminue avec l'augmentation de la dose en déjections de lapin. On note une différence significative $\left(\mathrm{F}_{(5,12)}=8,52 ; p<\right.$ 0,0012 ) entre les traitements (Tableaux 1 et 2). De même, il a été observé des variations pour la salinité (due aux déjections de lapin) et les solides totaux dissous (TDS) dans tous les traitements avec une différence très hautement significative (respectivement $\mathrm{F}_{(5,12)}=286,13 ; p<0,00$ et $\mathrm{F}_{(5,12)}=17,25 ; p<0,004$, Tableaux 1 et $\mathbf{2}$ ).

\subsection{Influence des nutriments des différentes doses de déjections sur la qualité de l'eau}

Les concentrations de $\mathrm{N}-\mathrm{NH}_{3}$ obtenues le jour d'ensemencement sont importantes dans tous les traitements sauf $\mathrm{T}_{0}$ et varient entre $0,03 \mathrm{mg} \cdot \mathrm{l}^{-1}\left(\mathrm{~T}_{0}\right)$ et $3,46 \mathrm{mg} \cdot \mathrm{l}^{-1} \quad\left(\mathrm{~T}_{5}\right)$ (Figure 2a). Ces différentes concentrations diminuent progressivement pour atteindre les valeurs minimales qui se situent entre $0,01 \mathrm{mg} \cdot \cdot^{-1}\left(\mathrm{~T}_{0}\right)$ et $2,02 \mathrm{mg} \cdot \mathrm{l}^{-1}\left(\mathrm{~T}_{5}\right)$ à la fin de l'expérimentation. En outre, dans le traitement $\mathrm{T}_{2}$, la quantité de $\mathrm{N}-\mathrm{NH}_{3}$ et celle de $\mathrm{P}-\mathrm{PO}_{4}{ }^{3-}$ de la deuxiè̀me semaine à la neuvième semaine sont respectivement de 0,05 à $0,21 \mathrm{mg} \cdot \mathrm{l}^{-1}$ et 0,7 à $1,65 \mathrm{mg} \cdot \mathrm{l}^{-1}$ (Figures 2a et 2d). Les quantités de $\mathrm{N}^{-\mathrm{NO}_{3}}$ se situent entre $0,01 \mathrm{mg} \cdot l^{-1}$ et $1,17 \mathrm{mg} \cdot \mathrm{l}^{-1}$ respectivement pour $\mathrm{T}_{0}$ et $\mathrm{T}_{5}$. Ces quantités obtenues sont importantes et 


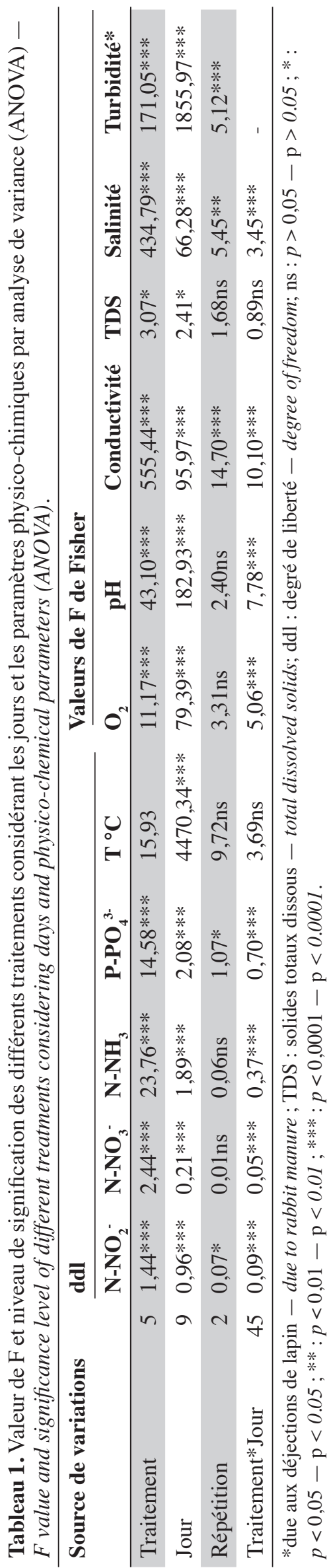

relativement constantes dans tous les seaux durant l'expérimentation (Figure 2b). Quant aux $\mathrm{N}_{-} \mathrm{NO}_{2}^{-}$, des concentrations élevées $\left(1,50\right.$ à $\left.1,45 \mathrm{mg} \cdot \mathrm{l}^{-1}\right)$ ont été enregistrées entre la semaine d'ensemencement et la première semaine, suivie d'une baisse notable dans tous les traitements $\left(0,03 \mathrm{mg} \cdot \mathrm{l}^{-1}\right)$. Les valeurs maximales ont été enregistrées dans les traitements $\mathrm{T}_{4}$ et $\mathrm{T}_{5}$ (Tableau 2 et Figure 2c), tandis que les concentrations minimales sont enregistrées dans les traitements $\mathrm{T}_{0}$ et $\mathrm{T}_{1}$ (Tableau 2). En ce qui concerne le $\mathrm{P}_{-} \mathrm{PO}_{4}^{3-}$, la concentration est maximale dans le traitement $\mathrm{T}_{5}$ et atteint $2,61 \mathrm{mg} \cdot \mathrm{l}^{-1}$ entre la première et la quatrième semaine d'expérimentation, suivie d'une chute significative à partir de la cinquième semaine dans tous les traitements (Figure 2d) $\left(\mathrm{F}_{(27,510)}=4,45\right.$; $p<0,00)$. En revanche, dans les traitements $\mathrm{T}_{2}$ et $\mathrm{T}_{3}$, elle est moins élevée et diminue par la suite au cours de l'expérimentation (Figure 2d) avec une évolution en dents de scie. Il n'existe pas de différence significative entre $\mathrm{T}_{1}, \mathrm{~T}_{2}$ et $\mathrm{T}_{3}\left(\mathrm{~F}_{(5,522)} ; p>0,05\right.$; Tableau 2). De manière globale, il existe une différence hautement significative des concentrations en $\mathrm{N}^{-\mathrm{NH}_{3}}, \mathrm{~N}^{-\mathrm{NO}_{3}}$, $\mathrm{N}-\mathrm{NO}_{2}^{-}, \mathrm{P}_{-} \mathrm{PO}_{4}^{3-}$ entre les traitements $\left(\mathrm{F}_{65,522)}=23,61^{\text {; }}\right.$ $p<0,00$, Tableau 1). Il ressort de cette analyse que le fertilisant organique (déjections de lapin) utilisé a fortement affecté la qualité de l'eau des différents milieux de production des macroinvertébrés benthiques d'eau douce, avec toutefois une influence variant selon la dose de déjections de lapin appliquée et selon le temps.

\subsection{Relation taille-poids et biomasse des Chironomidae et des Mollusques (Melanoides tuberculata)}

Les figures $\mathbf{3}$ et $\mathbf{4}$ illustrent les résultats obtenus concernant les analyses des régressions entre les poids secs et les longueurs, respectivement pour les Chironomidae de l'ordre des Diptères et $M$. tuberculata produits à partir des déjections de lapin. Elles montrent une corrélation linéaire entre le logarithme du poids sec et la longueur pour les Chironomidae $(r=0,91$ avec $p<0,05, \mathrm{n}=18)$ et pour les Mollusques $(\mathrm{r}=0,97$ avec $p<0,05 ; \mathrm{n}=18$ ) (Figures 3 et $\mathbf{4}$ ).

Les modèles de régressions obtenus respectivement pour les Chironomidae et les Mollusques sont :

$$
\begin{aligned}
& \mathrm{PS}_{(\mathrm{mg}) \text { Chironomidae }}=0,29 \mathrm{~L}^{0,93} \\
& \left(\mathrm{r}^{2}=0,84 ; \mathrm{F}_{(5,12)}=775,66 \text { avec } p<0,05\right) \\
& \mathrm{PS}_{(\mathrm{mg}) \text { Mollusque }}=1,14 \cdot 10^{-4} \mathrm{~L}^{4,19} \\
& \left(\mathrm{r}^{2}=0,94 ; \mathrm{F}_{(5,12)}=66,83 \text { avec } p<0,05\right)
\end{aligned}
$$


Tableau 2. Caractéristiques physico-chimiques des milieux de culture selon leur concentration en déjections de lapin Physico-chemical characteristics of culture media according to their content in rabbit manure.

\begin{tabular}{|c|c|c|c|c|c|c|}
\hline Paramètre & $\mathbf{T}_{0}$ & $\mathrm{~T}_{1}$ & $\mathbf{T}_{2}$ & $\mathbf{T}_{3}$ & $\mathbf{T}_{4}$ & $\mathbf{T}_{5}$ \\
\hline Température $\left({ }^{\circ} \mathrm{C}\right)$ & $31,57 \pm 0,58^{\mathrm{a}}$ & $31,53 \pm 0,60^{\mathrm{a}}$ & $31,51 \pm 0,63^{\mathrm{a}}$ & $31,46 \pm 0,61^{\mathrm{a}}$ & $31,53 \pm 0,60^{\mathrm{a}}$ & $31,59 \pm 0,60^{\mathrm{a}}$ \\
\hline $\mathrm{pH}$ & $7,40 \pm 0,11^{\mathrm{a}}$ & $7,24 \pm 0,11^{b}$ & $7,12 \pm 0,10^{c}$ & $7,08 \pm 0,11^{\mathrm{c}}$ & $6,95 \pm 0,11^{\mathrm{d}}$ & $6,84 \pm 0,10^{\mathrm{e}}$ \\
\hline Conductivité $\left(\mu \mathrm{s} \cdot \mathrm{cm}^{-1}\right)$ & $496,73 \pm 16,31^{\mathrm{e}}$ & $499,30 \pm 13,02^{\mathrm{e}}$ & $621,06 \pm 14,53^{\mathrm{d}}$ & $727,75 \pm 18,52^{\mathrm{c}}$ & $802,79 \pm 18,27^{b}$ & $874,21 \pm 30,58^{a}$ \\
\hline Oxygène (mg. $\left.\mathrm{l}^{-1}\right)$ & $4,11 \pm 0,12^{\mathrm{a}}$ & $3,23 \pm 0,29^{\mathrm{bc}}$ & $3,28 \pm 0,46^{\mathrm{b}}$ & $2,81 \pm 0,38^{\mathrm{cd}}$ & $2,99 \pm 0,45^{\mathrm{bcd}}$ & $2,70 \pm 0,40^{\mathrm{d}}$ \\
\hline TDS & $253,41 \pm 9,18^{c}$ & $349,19 \pm 91,36^{\mathrm{bc}}$ & $318,46 \pm 8,58^{\mathrm{bc}}$ & $370,92 \pm 9,37^{\mathrm{ab}}$ & $374,93 \pm 31,46^{\mathrm{ab}}$ & $461,01 \pm 10,65^{\mathrm{a}}$ \\
\hline Salinité (mg. $\mathrm{l}^{-1}$ ) & $0,27 \pm 0,00^{\mathrm{e}}$ & $0,27 \pm 0,00^{\mathrm{e}}$ & $0,34 \pm 0,00^{\mathrm{d}}$ & $0,39 \pm 0,00^{c}$ & $0,44 \pm 0,00^{\mathrm{b}}$ & $0,48 \pm 0,01^{\mathrm{a}}$ \\
\hline Turbidité (NTU) & $28,11 \pm 2,00^{\mathrm{e}}$ & $48,26 \pm 1,68^{e}$ & $84,77 \pm 4,01^{\mathrm{d}}$ & $130,03 \pm 11,73^{c}$ & $167,07 \pm 26,25^{b}$ & $275,77 \pm 4,63^{a}$ \\
\hline $\mathrm{N}-\mathrm{NH}_{3}\left(\mathrm{mg} \cdot l^{-1}\right)$ & $0,02 \pm 0,00^{\mathrm{f}}$ & $0,56 \pm 0,09^{\mathrm{e}}$ & $0,98 \pm 0,09^{\mathrm{d}}$ & $1,62 \pm 0,03^{\mathrm{c}}$ & $1,88 \pm 0,07^{\mathrm{b}}$ & $2,42 \pm 0,13^{\mathrm{a}}$ \\
\hline $\mathrm{N}-\mathrm{NO}_{2}^{-}\left(\mathrm{mg} \cdot \mathrm{l}^{-1}\right)$ & $0,03 \pm 0,00^{\mathrm{e}}$ & $0,38 \pm 0,07^{b}$ & $0,29 \pm 0,05^{\mathrm{c}}$ & $0,33 \pm 0,04^{\mathrm{bc}}$ & $0,38 \pm 0,03^{b}$ & $0,71 \pm 0,07^{\mathrm{a}}$ \\
\hline $\mathrm{N}-\mathrm{NO}_{3}^{-}\left(\mathrm{mg} \cdot \mathrm{l}^{-1}\right)$ & $0,04 \pm 0,01^{\mathrm{d}}$ & $0,16 \pm 0,01^{\mathrm{c}}$ & $0,19 \pm 0,01^{\mathrm{c}}$ & $0,19 \pm 0,01^{\mathrm{c}}$ & $0,57 \pm 0,04^{\mathrm{b}}$ & $0,78 \pm 0,05^{\mathrm{a}}$ \\
\hline $\mathrm{P}_{-}-\mathrm{PO}_{4}^{3-}\left(\mathrm{mg} \cdot \mathrm{l}^{-1}\right)$ & $0,46 \pm 0,03^{d}$ & $1,29 \pm 0,14^{\mathrm{bc}}$ & $1,21 \pm 0,11^{\mathrm{c}}$ & $1,17 \pm 0,12^{\mathrm{c}}$ & $1,43 \pm 0,05^{\mathrm{b}}$ & $2,61 \pm 0,16^{\mathrm{a}}$ \\
\hline
\end{tabular}

Les traitements $\mathrm{T}_{0}, \mathrm{~T}_{1}, \mathrm{~T}_{2}, \mathrm{~T}_{3}, \mathrm{~T}_{4}$ et $\mathrm{T}_{5}$ ont été fertilisés respectivement avec $0 \%, 10 \%, 25 \%, 50 \%, 75 \%$ et $100 \%$ de déjections de lapin par rapport au volume total du substrat - the treatments $T_{0}, T_{1}, T_{2}, T_{3}, T_{4}$ and $T_{5}$ were respectively fertilized with $0 \%, 10 \%, 25 \%, 50 \%$, $75 \%$ and $100 \%$ of rabbit manure relative to the total volume of the substrate; les valeurs moyennes affectées d'une même lettre sur la même ligne ne sont pas significativement différentes au seuil de $5 \%$ selon LSD - the means with the same letter on the same line are not significantly different at the treshold $5 \%$ according to LSD.

a
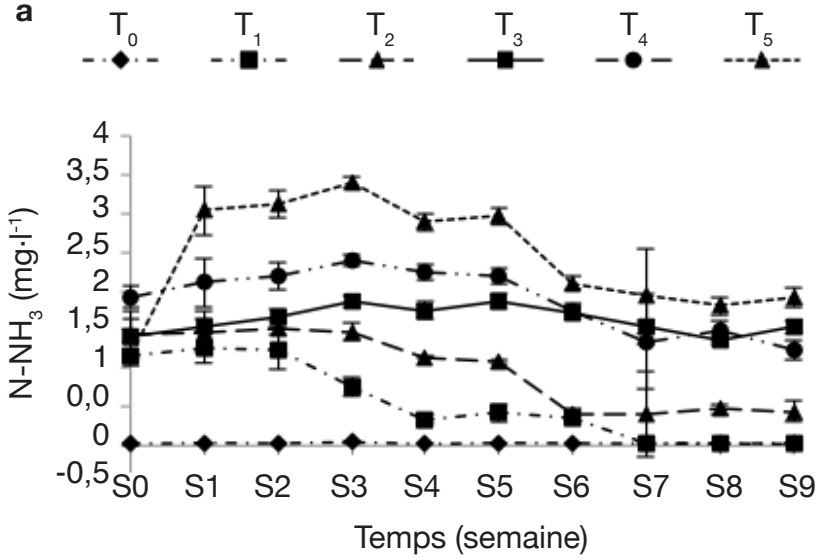

C

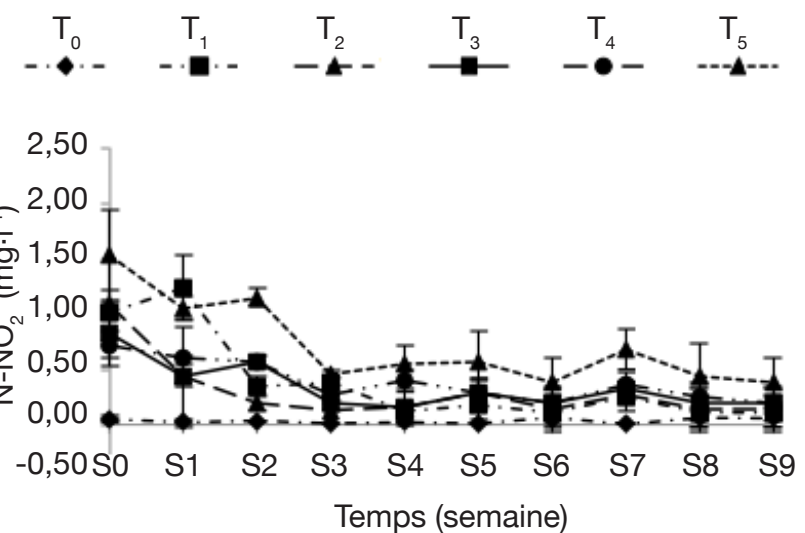

b

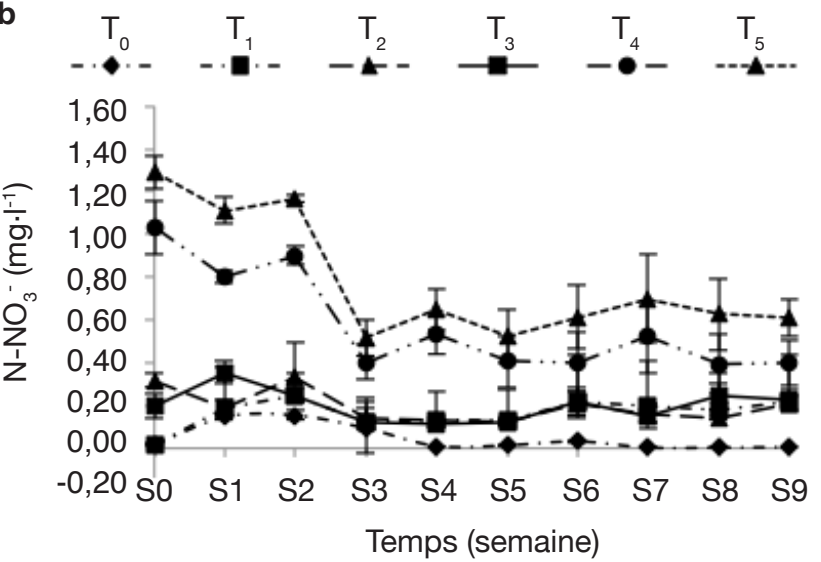

d

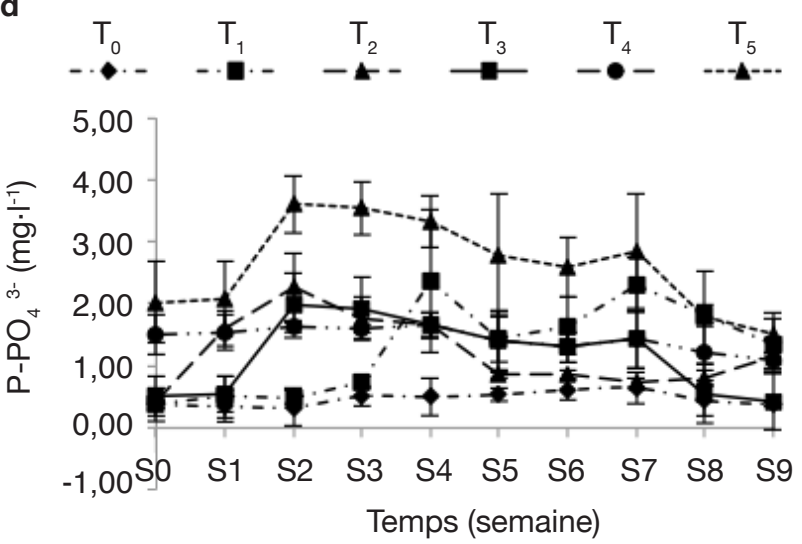

Figure 2. Évolution des paramètres chimiques en fonction des concentrations de déjections de lapin - Evolution of chemical parameters with rabbit manure content.

$\mathrm{T}$ : traitement - treatment, voir tableau 2 - see table 2. 


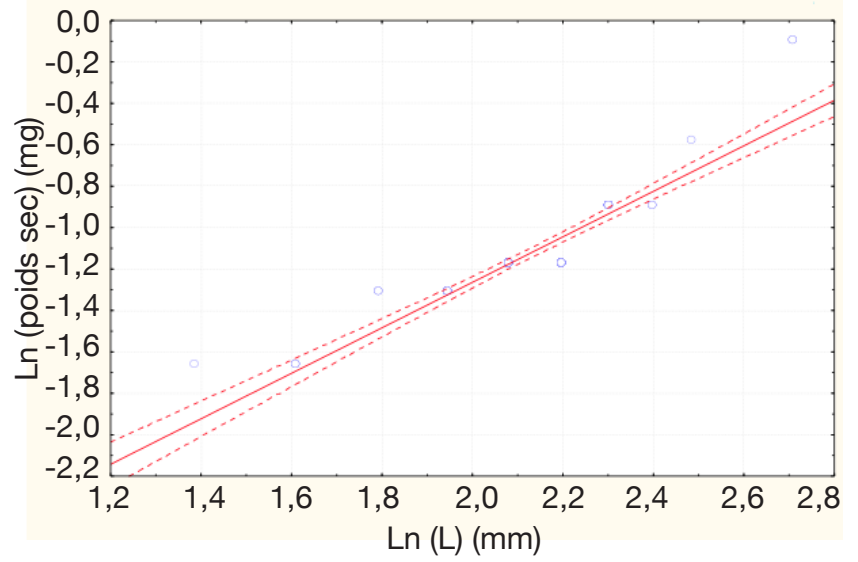

Figure 3. Relation taille-poids des Chironomidae (Diptère, Nématocère) - Size-weight relationship of Chironomidae (Diptera, Nematocera).

$\mathrm{r}^{2}=0,84 ; \mathrm{r}=0,91 ; p=0,00 ; \mathrm{y}=-3,46 \times 1,10^{*} \mathrm{x} ; \mathrm{Ic}=0,95$.

Les valeurs des coefficients obtenus sont significativement différentes de 0 (valeurs de $t$ obtenues avec $p<0,05)$.

Les écarts entre les poids secs observés et ceux estimés à l'aide de ces équations n'étant pas significativement différents de 0 , ces équations ne nécessitent pas d'appliquer des facteurs de correction pour une bonne estimation des biomasses.

\subsection{Influence de la dose de déjections sur la biomasse et la densité des macroinvertébrés benthiques}

Les biomasses des Chironomidae et des Mollusques des différents traitements ont été estimées à partir des deux relations taille-poids précédemment établies. La comparaison des biomasses obtenues par traitement révèle une différence significative aussi bien pour les Chironomidae $\left(\mathrm{F}_{(5,12)}=775,6 ; p<0,05 ; \mathrm{n}=18\right)$ que pour les Mollusques $\left(\mathrm{F}_{(5,12)}=66,8, p<0,05, \mathrm{n}=\right.$ 18). La biomasse la plus élevée des Chironomidae et des Mollusques est obtenue pour $\mathrm{T}_{2}$ (Figure 5) avec respectivement $728,1 \pm 2,23 \mathrm{mg}$ de matière sèche $\mathrm{dm}^{-2}$ et $699,9 \pm 22,4 \mathrm{mg}$ de matière sèche $\cdot \mathrm{dm}^{-2}$ soit $364 \pm 2,2$ et $349,9 \pm 22,4 \mathrm{mg}$ de matière sèche $\cdot \mathrm{dm}^{-3}$ de Chironomidae et Mollusques, respectivement. Elle est suivie de $\mathrm{T}_{4}, \mathrm{~T}_{3}, \mathrm{~T}_{1}, \mathrm{~T}_{5}, \mathrm{~T}_{0}$ avec respectivement $347,4 \pm 3 \mathrm{mg}$ de matière sèche $\cdot \mathrm{dm}^{-2}, 268,8 \pm 9,2 \mathrm{mg}$ de matière sèche $\cdot \mathrm{dm}^{-2}, 263,5 \pm 3,9 \mathrm{mg}$ de matière sèche. $\mathrm{dm}^{-2}, 84,1 \pm 2,4 \mathrm{mg}$ de matière sèche $\cdot \mathrm{dm}^{-2}$ et $16,1 \pm$ $0,1 \mathrm{mg}$ de matière sèche $\cdot \mathrm{dm}^{-2}$ (soit $173,7 \pm 3 \mathrm{mg}$ de matière sèche $\cdot \mathrm{dm}^{-3}, 134,4 \pm 9,2 \mathrm{mg}$ de matière sèche . $\mathrm{dm}^{-3}, 131,7 \pm 3,9 \mathrm{mg}$ de matière sèche $\cdot \mathrm{dm}^{-3}, 42 \pm 2,4$ $\mathrm{mg}$ de matière sèche $\cdot \mathrm{dm}^{-3}$ et $8 \pm 0,1 \mathrm{mg}$ de matière sèche $\cdot \mathrm{dm}^{-3}$ ) avec une différence significative entre les biomasses $(p<0,05)$ de Chironomidae. Mais chez les

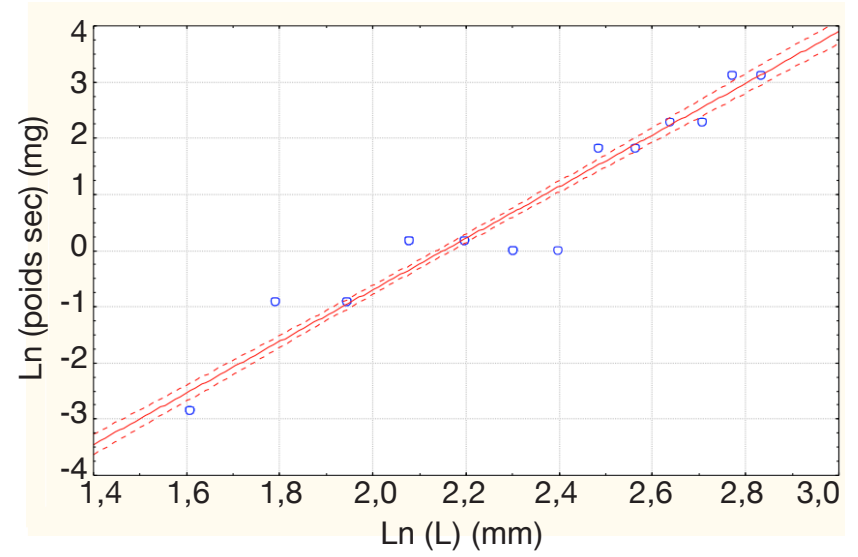

Figure 4. Relation taille-poids des Mollusques d'eau douce Melanoides tuberculata - Size-weight relationship of freshwater mollusks Melanoides tuberculata.

$\mathrm{r}^{2}=0,94 ; \mathrm{r}=0,97 ; p=0,00 ; \mathrm{y}=-9,89+4,59 * \mathrm{x} ; \mathrm{Ic}=0,95$.

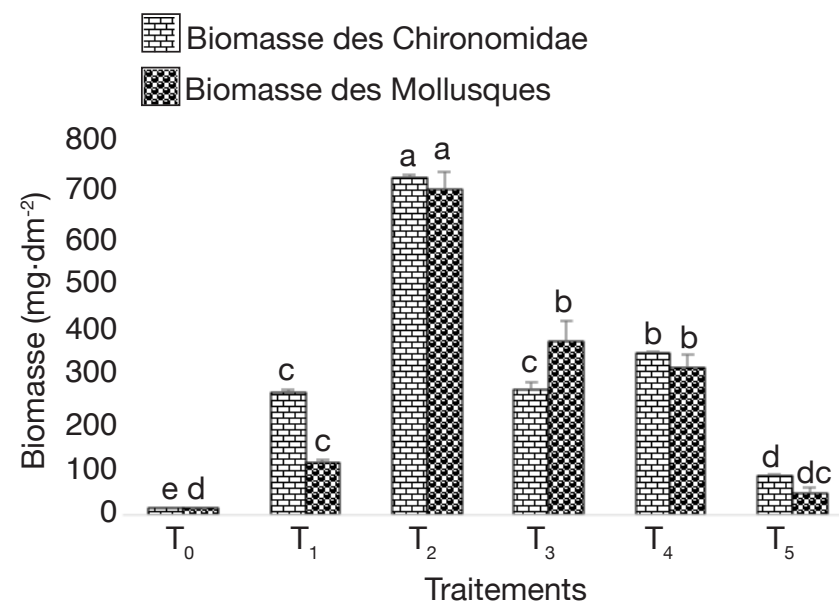

Figure 5. Biomasses des Chironomidae et des Mollusques des différents traitements - Chironomid and mollusks biomass of different treatments.

$\mathrm{T}$ : traitement - treatment, voir tableau 2 - see table 2; les biomasses possédant les mêmes lettres ne sont pas significativement différentes au seuil $\alpha=5 \%$ - the biomasses with the same letters are not significantly different at the treshold $\alpha=5 \%$.

Mollusques, la biomasse maximale enregistrée dans $\mathrm{T}_{2}$ est suivie de $\mathrm{T}_{3}\left(375 \pm 25,2 \mathrm{mg}\right.$ de matière sèche $\cdot \mathrm{dm}^{-2}$ ou $187,5 \pm 25,2 \mathrm{mg}$ de matière sèche $\left.\cdot \mathrm{dm}^{-3}\right), \mathrm{T}_{4}(316,4 \pm$ $17,3 \mathrm{mg}$ de matière sèche $\cdot \mathrm{dm}^{-2}$ ou $158,2 \pm 17,3 \mathrm{mg}$ de matière sèche $\left.\cdot \mathrm{dm}^{-3}\right), \mathrm{T}_{1}(113,1 \pm 4,2 \mathrm{mg}$ de matière sèche $\cdot \mathrm{dm}^{-2}$ ou $56,5 \pm 4,2 \mathrm{mg}$ de matière sèche $\left.\cdot \mathrm{dm}^{-3}\right), \mathrm{T}_{5}$ $\left(47,9 \pm 7,1 \mathrm{mg}\right.$ de matière sèche $\cdot \mathrm{dm}^{-2}$ ou $23,9 \pm 7,1 \mathrm{mg}$ de matière sèche $\left.\cdot \mathrm{dm}^{-3}\right)$ et $\mathrm{T}_{0}(14,4 \pm 0,5 \mathrm{mg}$ de matière sèche $\cdot \mathrm{dm}^{-2}$ ou $7,2 \pm 0,5 \mathrm{mg}$ de matière sèche $\cdot \mathrm{dm}^{-3}$ ) avec une différence significative entre les biomasses $(p<0,05)$ (Figure 5). 
Durant la période d'expérimentation, quatre groupes de macroinvertébrés ont été dénombrés, il s'agit des Annélides, des Chironomidae (vers de sang), des Odonates et des Mollusques (Melanoides tuberculata) (Figure 6). Les Chironomidae et les

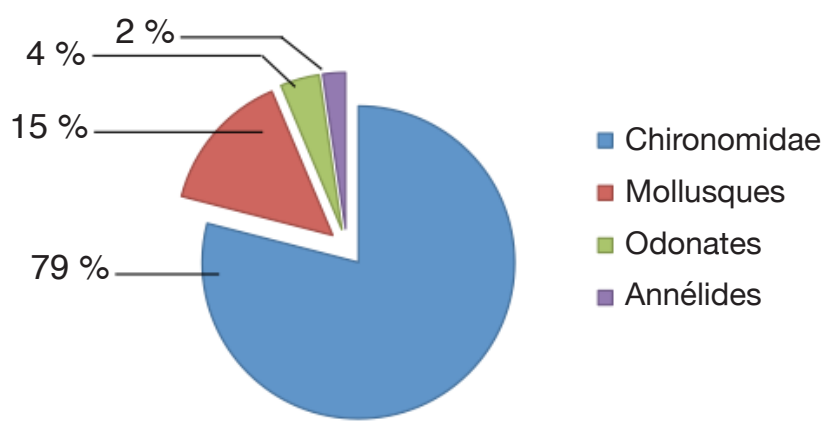

Figure 7. Proportions des groupes de macroinvertébrés benthiques d'eau douce issus des différents milieux de production - Proportions of freshwater benthic macroinvertebrates groups from different production media.

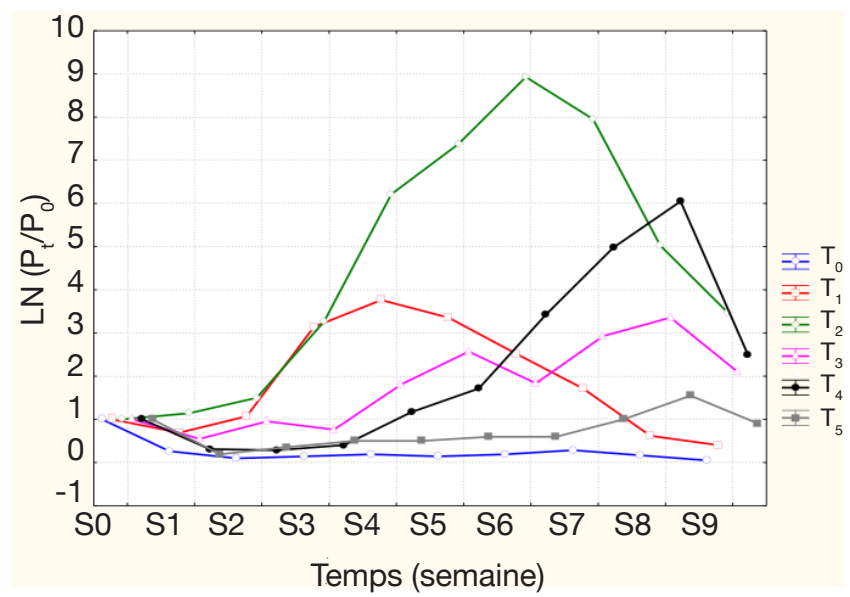

Figure 8. Évolution de la biomasse relative selon les traitements voir tableau $2-$ Evolution of relative biomass according to treatments (see table 2).
Mollusques ont été les taxons dominants au cours de la production, suivis des Odonates et Annélides (Figure 7). Quelle que soit la dose de déjections de lapin apportée, la densité de l'ensemble des macroinvertébrés suit la même évolution temporelle que la biomasse (Figure 8) entre la $1^{\text {ère }}$ et la $9^{\mathrm{e}}$ semaine avec trois pics les $4^{\mathrm{e}}, 6^{\mathrm{e}}$ et $8^{\mathrm{e}}$ semaines respectivement pour $\mathrm{T}_{1}, \mathrm{~T}_{2}$ et $\mathrm{T}_{4}$. Lors des différents pics, la densité la plus élevée est obtenue avec le traitement $\mathrm{T}_{2}$ suivi de $\mathrm{T}_{4}$ et $\mathrm{T}_{1}$. Toutefois, pendant cette période, l'évolution de la densité diffère selon les groupes de macroinvertébrés et les doses de déjections de lapin. Pour $\mathrm{T}_{2}$, elle est de $192 \pm 1,2$ ind $\cdot \mathrm{dm}^{-2}$ de Chironomidae, $52 \pm 0,8$ ind. $\mathrm{dm}^{-2}$ de Mollusques, $6 \pm 0,3$ ind $\cdot \mathrm{dm}^{-2} \mathrm{~d}$ 'Odonates et $4 \pm$ 0,3 ind $\cdot \mathrm{dm}^{-2} \mathrm{~d}$ 'Annélides en fin d'expérience, soit pour $\mathrm{T}_{2}, 96 \pm 1,2$ ind $\cdot \mathrm{dm}^{-3}$ de Chironomidae, $26 \pm 0,8$ ind $\mathrm{dm}^{-3}$ de Mollusques, $3 \pm 0,3$ ind $\cdot \mathrm{dm}^{-3}$ d'Odonates et $2 \pm 0,3$ ind $\cdot \mathrm{dm}^{-3}$ d'Annélides. La densité moyenne des Chironomidae pour toute la période est plus élevée pour le traitement $T_{2}\left(104 \pm 6,2\right.$ ind $\cdot \mathrm{dm}^{-2}$, soit $52 \pm$ 6,2 ind $\cdot \mathrm{dm}^{-3}$ ). Elle est suivie de $\mathrm{T}_{4}, \mathrm{~T}_{1}, \mathrm{~T}_{5}$ et $\mathrm{T}_{0}$ avec respectivement $52 \pm 5,2 \mathrm{ind} \cdot \mathrm{dm}^{-2}, 40 \pm 2,7 \mathrm{ind} \cdot \mathrm{dm}^{-2}$, $14 \pm 1$ ind $\cdot \mathrm{dm}^{-2}$ et $2 \pm 0,2$ ind $\cdot \mathrm{dm}^{-2}$ (soit $26 \pm 5,2$ ind. $\mathrm{dm}^{-3}, 20 \pm 2,7$ ind $\cdot \mathrm{dm}^{-3}, 7 \pm 1$ ind $\cdot \mathrm{dm}^{-3}$ et $1 \pm 0,2$ ind. $\left.\mathrm{dm}^{-3}\right)$ avec une différence significative $(p<0,05)$. Pour les Mollusques, la densité moyenne est de $20 \pm$ 1,2 ind $\cdot \mathrm{dm}^{-2}$ (soit $10 \pm 1,2$ ind $\cdot \mathrm{dm}^{-3}$ ) pour $\mathrm{T}_{2}$ suivi de $8 \pm 0,4$ ind $\cdot \mathrm{dm}^{-2}, 6 \pm 0,4$ ind $\cdot \mathrm{dm}^{-2}, 6 \pm 0,4$ ind $\cdot \mathrm{dm}^{-2}, 3 \pm$ 0,2 ind $\cdot \mathrm{dm}^{-2}, 2 \pm 0,2$ ind $\cdot \mathrm{dm}^{-2}$ soit $\left(4 \pm 0,4 \mathrm{ind} \cdot \mathrm{dm}^{-3}\right.$, $3 \pm 0,4$ ind $\cdot \mathrm{dm}^{-3}, 3 \pm 0,4$ ind $\cdot \mathrm{dm}^{-3}, 3 \pm 0,2 \mathrm{ind} \cdot \mathrm{dm}^{-3}$, $1 \pm 0,2$ ind $\left.\cdot \mathrm{dm}^{-3}\right)$ respectivement de $\mathrm{T}_{1}, \mathrm{~T}_{3}, \mathrm{~T}_{4}, \mathrm{~T}_{5}, \mathrm{~T}_{0}$ et significativement différente $(p<0,05)$. On note une faible densité moyenne des Odonates et des Annélides dans tous les traitements variant entre 1 et 2 . Toutefois, le traitement $\mathrm{T}_{2}$ affiche la densité la plus élevée.
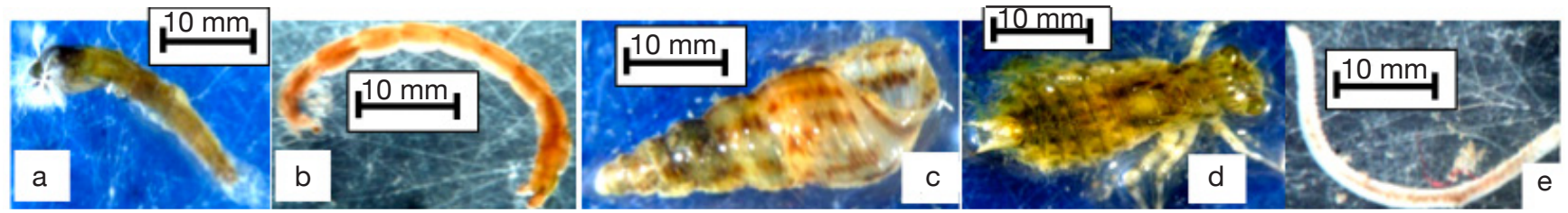

Figure 6. Photographie de macroinvertébrés d'eau douce issus de la culture en batch. a: nymphe de Chironomus sp., b: Chironomus sp. adulte (Chironomidae), c: Melanoides tuberculata (Mollusque, Gastéropode), d: Odonate sp. (Anisoptère, Libellulidae), e: Naïdidae sp. (Annélides, Oligochète) - Photograph of freshwater macroinvertebrates from batch culture. $\boldsymbol{a}$ : Chironomus sp. (Chironomidae), nymph, b: Chironomus sp. (Chironomidae), adult, $\boldsymbol{c}$ : Melanoides tuberculata (Mollusca, Gastropoda), d: Odonate sp. (Anisoptera, Libellulidae), e: Naïdidae sp. (Annelida Oligochaeta). 


\subsection{Effet des doses de déjections sur la vitesse de croissance des macroinvertébrés benthiques}

L'évolution globale du logarithme de la biomasse relative des macroinvertébrés observée durant l'expérimentation dans chaque traitement est illustrée sur la figure 8.

Les taux de croissance spécifiques, déterminés pour les différents traitements sur la phase de croissance exponentielle, sont présentés dans le tableau 3. Il existe une différence hautement significative entre les taux de croissances spécifiques des différents traitements $\left(\mathrm{F}_{(5,12)}=1361 ; p<0,00, \mathrm{n}=18\right)$. De la comparaison multiple de l'effet des traitements (test LSD de Fisher), il ressort que $\mathrm{T}_{2}$ suivi de $\mathrm{T}_{4}$ présentent un meilleur taux de croissance spécifique.

La vitesse d'augmentation de la biomasse de Chironomidae dans les milieux fertilisés avec des déjections de lapin est estimée à $5,26 \mathrm{mg}$ de matière sèche $\cdot \mathrm{dm}^{-2} \cdot \mathrm{j}^{-1}$ (soit $2,6 \mathrm{mg}$ de matière sèche $\cdot \mathrm{dm}^{-3} \cdot \mathrm{j}^{-1}$ ) contre $0,2 \mathrm{mg}$ de matière sèche $\cdot \mathrm{dm}^{-2} \cdot \mathrm{j}^{-1}$ (soit $0,1 \mathrm{mg}$. $\mathrm{dm}^{-3} \cdot \mathrm{j}^{-1}$ ) dans le milieu non fertilisé ; pour les Mollusques, elle est de $4,9 \mathrm{mg}$ de matière sèche $\cdot \mathrm{dm}^{-2} \cdot \mathrm{j}^{-1}$ (soit 2,4 mg de matière sèche $\cdot \mathrm{dm}^{-3} \cdot \mathrm{j}^{-1}$ ) dans les milieux fertilisés, contre $0,2 \mathrm{mg}$ de matière sèche $\cdot \mathrm{dm}^{-2} \cdot \mathrm{j}^{-1}$ (soit $0,1 \mathrm{mg}$ de matière sèche $\left.\cdot \mathrm{dm}^{-3} \cdot \mathrm{j}^{-1}\right)$ dans le milieu non fertilisé.

\section{DISCUSSION}

\subsection{Qualité physico-chimique du milieu de production}

Le développement des macroinvertébrés benthiques dépend inévitablement des conditions qu'offrent les

Tableau 3. Taux de croissance spécifique des macroinvertébrés dans les différents traitements - Specific growth rate of macroinvertebrates in different treatments.

\begin{tabular}{lll}
\hline Traitement & $\begin{array}{l}\text { Taux de croissance spécifique }(\boldsymbol{\mu}) \\
\text { (par semaine) }\end{array}$ & $\mathbf{R}^{\mathbf{2}}$ \\
\hline $\mathrm{T}_{0}$ & $0,07^{\mathrm{f}}$ & 0,96 \\
$\mathrm{~T}_{1}$ & $1,13^{\mathrm{c}}$ & 0,93 \\
$\mathrm{~T}_{2}$ & $1,82^{\mathrm{a}}$ & 0,96 \\
$\mathrm{~T}_{3}$ & $0,36^{\mathrm{e}}$ & 0,70 \\
$\mathrm{~T}_{4}$ & $1,31^{\mathrm{b}}$ & 0,96 \\
$\mathrm{~T}_{5}$ & $0,33^{\mathrm{d}}$ & 0,87 \\
\hline
\end{tabular}

$\mathrm{T}$ : traitement - treatment, voir tableau 2 - see table 2; les valeurs moyennes affectées d'une même lettre ne sont pas significativement différentes au seuil $\alpha=5 \%$ selon LSD - the means with a same letter are not significantly different at the treshold $\alpha=5 \%$ according to LSD. paramètres physico-chimiques et trophiques du milieu. Ainsi, l'effet de la fertilisation sur le développement de la biomasse des macroinvertébrés benthiques est bien démontré aux doses $\mathrm{T}_{1}, \mathrm{~T}_{2}, \mathrm{~T}_{3}$ et $\mathrm{T}_{4}$. La température enregistrée au cours de cette étude est de $31 \pm 0,6^{\circ} \mathrm{C}$. Elle est proche de celle enregistrée par Sulistiyarto \& Yulintine (2014) au cours de la production des Chironomidae. En effet, selon Tokeshi (1995), Angelier (2003) et Hussain \& Pandit (2012), la température affecte le taux de croissance et le métabolisme des macroinvertébrés benthiques. Cette température est largement supérieure à celle enregistrée par Maleknejad et al. (2014) qui est de $26{ }^{\circ} \mathrm{C}$. Cette différence pourrait être due à la saison et au climat dans lesquels les expériences se sont déroulées. La gamme de tolérance du $\mathrm{pH}$ des macroinvertébrés est comprise entre 5,5 et 8,4 (Nath, 2014). Ainsi, le pH des différents traitements $(6,95-7,24)$ offre des conditions favorables de développement. Le $\mathrm{pH}$ et l'oxygène dissous des traitements $\mathrm{T}_{0}, \mathrm{~T}_{1}$ et $\mathrm{T}_{2}$ sont comparables aux résultats enregistrés sur la rivière São Lourenço au Brésil (Rosa et al., 2014). En revanche, les valeurs élevées de conductivité, de solides totaux dissous (TDS), de la turbidité corrélée avec une faible valeur d'oxygène dans les traitements $\mathrm{T}_{3}, \mathrm{~T}_{4}, \mathrm{~T}_{5}$ sont dues à des quantités élevées de déjections apportées. En effet, selon Shortle et al. (2001), une quantité élevée de matière organique diminue l'oxygène et augmente parallèlement la turbidité et la conductivité. De même, Cunha-Santino $\&$ Bianchini (2003), Kelly et al. (2013) et Rosa et al. (2014) signalent qu'une quantité élevée de matière organique pourrait occasionner une augmentation de la demande biochimique en oxygène (DBO) et du carbone organique total (COT: carbone organique dissous et particulaire) ainsi qu'une diminution du développement des algues. Ces conditions semblent affecter la densité des macroinvertébrés dans les traitements $\mathrm{T}_{3}, \mathrm{~T}_{4}, \mathrm{~T}_{5}$, contrairement au $\mathrm{T}_{2}$ qui offre de très bonnes conditions de développement. En effet, une charge très élevée en matière organique des milieux aquatiques affecte la biomasse des macroinvertébrés (de Meutter et al., 2005). Les différentes concentrations moyennes de salinité (due aux déjections de lapin) obtenues avec les différents traitements sont bien comprises entre 0 et $5 \mathrm{mg} \cdot \mathrm{l}^{-1}$, valeurs signalées par Cartier et al. (2011) pour une bonne production de macroinvertébrés benthiques. Selon les travaux de Wang et al. (2007), la concentration optimale pour la survie et une bonne croissance des Mollusques est comprise entre 0,006 et $0,12 \mathrm{mg} \cdot l^{-1}$ de $\mathrm{N}-\mathrm{NH}_{3}$ et est proche de celles enregistrées avec la dose $\mathrm{T}_{2}\left(0,05-0,21 \mathrm{mg} \cdot \mathrm{l}^{-1} \mathrm{de}\right.$ $\mathrm{N}-\mathrm{NH}_{3}$ ) dans le cas de nos travaux, ce qui pourrait expliquer la biomasse des Mollusques obtenue avec ce traitement. Les valeurs de $\mathrm{N}^{-\mathrm{NO}_{3}}{ }^{-}\left(0,01-1,17 \mathrm{mg} \cdot \mathrm{l}^{-1}\right)$ enregistrées dans le cadre de cette étude sont proches de celles recommandées par Carmago et al. (2005) 
( $2 \mathrm{mg} \cdot \mathrm{l}^{-1}$ de $\mathrm{N}-\mathrm{NO}_{3}^{-}$) pour les invertébrés d'eau douce. En effet, cette concentration serait appropriée pour protéger les invertébrés d'eau douce les plus sensibles. Selon Passel et al. (2007), certains macroinvertébrés peuvent supporter des quantités de $\mathrm{N}_{-} \mathrm{NH}_{3}$ élevées dans les eaux douces dont le $\mathrm{pH}$ est égal à 7,5. En effet, la présence des macroinvertébrés dans les autres traitements dont la quantité de $\mathrm{N}-\mathrm{NH}_{3}$ est plus élevée pourrait être due au $\mathrm{pH}$ enregistré dans nos différents traitements. En outre, la forme de $\mathrm{NH}_{3}$ (toxique) est dominante à $50 \%$ à un $\mathrm{pH}$ supérieur à 9,3 à $20^{\circ} \mathrm{C}$, tandis qu'à un $\mathrm{pH}$ inférieur à 9,3 à la même température $\mathrm{NH}_{4}{ }^{+}$(non toxique) est dominante (Weiner, 2012). De plus, une concentration supérieure à $0,5 \mathrm{mg} \cdot \mathrm{l}^{-1}$ de $\mathrm{N}-\mathrm{NH}_{3}$ serait significativement toxique pour les poissons (Weiner, 2012). Une concentration supérieure à $0,3 \mathrm{mg} \cdot 1^{-1}$ de phosphore montre la richesse de l'eau douce en nutriments propices au bon développement des macroinvertébrés benthiques (Struijs et al., 2010). Mais une quantité supérieure à $3,5 \mathrm{mg} \cdot \mathrm{l}^{-1}$ de phosphore provoquerait la disparition de la moitié des organismes benthiques avec le phénomène d'eutrophisation (Struijs et al., 2010). Dans cette expérience, les concentrations d'orthophosphate enregistrées dans le traitement $\mathrm{T}_{5}$ sont proches de $3,5 \mathrm{mg} \cdot \mathrm{l}^{-1}$, ce qui justifierait les faibles biomasses obtenues, et pourraient être dues à l'eutrophisation de ce milieu. Ainsi, en termes de nutriment, le traitement $\mathrm{T}_{2}$ offre donc un milieu favorable au bon développement des macroinvertébrés benthiques.

\subsection{Production de macroinvertébrés d'eau douce}

La baisse de densité par rapport à la densité initiale d'ensemencement enregistrée au cours de la première semaine (phase de latence) pourrait être due à la mort et à l'adaptation physiologique des organismes au milieu. Le traitement $\mathrm{T}_{2}$ dont la dose est de $25 \%$ de substrat (140 $\mathrm{g}$ de matière sèche de déjections de lapin $\cdot \mathrm{dm}^{-2} \mathrm{ou}$ $75 \mathrm{~g} \cdot \mathrm{dm}^{-3}$ de déjections de lapin) a donné la meilleure production de macroinvertébrés benthiques en termes de densité, de biomasse et de taux de croissance spécifique. Parmi les macroinvertébrés produits dans ce traitement, les Chironomidae représentent près de $79 \%$ des individus, suivis par les Mollusques (15\%). Ces résultats sont similaires à ceux de Matèna et al. (2016) qui ont obtenu $70 \%$ de Chironomidae, $20 \%$ de Ceratopogonidae et $10 \%$ des autres macroinvertébrés benthiques. Cette dominance des Chironomidae est un atout pour la pisciculture vu leurs qualités nutritionnelles pour les poissons (Shaw \& Mark,1980). Les travaux de Maleknejad et al. (2014) et Matèna et al. (2016) ont montré l'impact positif des fertilisants organiques sur la production de macroinvertébrés. De même, les résultats des travaux de Matèna et al. (2016) ont révélé, comme dans cette étude, que l'apport des bouses de vache améliore l'abondance des macroinvertébrés benthiques dans les étangs. Selon Thomaz et al. (2008) et Epele et al. (2012), la diversité et l'abondance des Chironomidae dépendent des aliments (essentiellement les algues et les détritus) et de la stabilité du substrat. De même, l'utilisation de déjections de lapin comme fertilisant organique permet un bon développement des macroinvertébrés comme des Chironomidae (vers de sang) et Mollusques via la production primaire et les détritus, comme le dénotent les résultats des travaux de Sanseverino \& Nessimian (2008) et Leite-Rossi \& Trivinho-Strixino (2012). La biomasse des Chironomidae obtenue dans le traitement $\mathrm{T}_{2}$ est toutefois supérieure à celle obtenue par Sulistiyarto \& Yulintine (2014) et comprise entre 7,2 et $102,9 \mathrm{mg} \cdot \mathrm{mg}^{-1}$ de substrat. La production moyenne obtenue avec $\mathrm{T}_{2}$ permet de multiplier au moins deux fois celle obtenue individuellement dans les traitements $\mathrm{T}_{1}, \mathrm{~T}_{3}$ et $\mathrm{T}_{4}$, six fois celle obtenue dans le traitement $\mathrm{T}_{5}$ et 18 fois celle observée dans le traitement $\mathrm{T}_{0}$ en termes de densité. De plus, la vitesse de croissance relative (A) (par semaine) des macroinvertébrés du traitement $\mathrm{T}_{2}$ est respectivement 1,$38 ; 1,61 ; 5,05 ; 5,51$ et 26 fois supérieure à celle observée dans les traitements $\mathrm{T}_{4}, \mathrm{~T}_{1}, \mathrm{~T}_{3}, \mathrm{~T}_{5}$ et $\mathrm{T}_{0}$. Très peu d'information existe dans la littérature concernant la vitesse de croissance des macroinvertébrés benthiques produits en milieu non renouvelé. Cependant, les différentes phases de croissances des macroinvertébrés sont identiques à celles obtenues pour les cultures microbiennes et, logiquement, seule une différence en termes d'ordre de grandeur du temps est observée (semaine) par rapport aux cultures microbiennes (généralement minutes ou heures).

En outre, les biomasses des Chironomidae et des Mollusques, dont la taille est comprise respectivement entre 4-12 mm et 5-21 mm, sont estimées à partir des régressions taille-poids. Les régressions obtenues sont fortement significatives et ont expliqué $84 \%$ et $94 \%$ de la variabilité totale, respectivement pour les Chironomidae et pour les Mollusques, avec des différences non significatives observées entre les biomasses mesurées et celles estimées à l'aide des modèles. Cependant, la précision de la balance n'est que $0,1 \mathrm{mg}$ dans le cadre de ce travail. Il est possible que les deux modèles issus des régressions puissent être utilisés pour estimer les biomasses de ces organismes produits à partir des déjections de lapin. Pour ces deux specimens, les valeurs de $b$ sont respectivement de 0,93 et 4,19 pour les Chironomidae et les Mollusques. Comme l'ont souligné Benke et al. (1999) et Miserendino (2001), pour $b$ supérieur à 3, le poids augmente plus vite que la longueur. En revanche, nos résultats sont différents de ceux obtenus par ces mêmes auteurs. Cette différence pourrait être due à la nature du fertilisant, du conservateur, du temps et du 
taxon (Miserendino, 2001). En effet, la production de macroinvertébrés benthiques à l'aide des déjections de porc $\left(742 \pm 569\right.$ ind $\left.\cdot \mathrm{dm}^{-3}\right)$, obtenue par Akodobgo et al. (2016), est largement supérieure à celle observée dans la présente expérience.

\section{CONCLUSIONS}

Les résultats de cette étude apportent une clarification de l'importance d'une utilisation rationnelle des fertilisants organiques pour assurer une bonne production piscicole. Dans le cas des déjections de lapin qui ont été ici étudiées, après avoir été produites à partir d'un régime alimentaire de composition bien connue, le traitement $\mathrm{T}_{2}$ qui correspond à une dose de $140 \mathrm{~g}$ de matière sèche de déjections $\cdot \mathrm{dm}^{-2}$ (ou $75 \mathrm{~g}$ de matière sèche de déjections $\cdot \mathrm{dm}^{-3}$ ) et le traitement $T_{4}$ qui correspond à une dose de $418 \mathrm{~g}$ de matière sèche de déjections de lapin. $\mathrm{dm}^{-2}$ (soit $225 \mathrm{~g}$ de matière sèche de déjections $\cdot \mathrm{dm}^{-3}$ ) offrent un milieu favorable pour un bon développement des macroinvertébrés benthiques d'eau douce. Ces deux traitements présentent de meilleures vitesses de croissance relative, densité et biomasse. En pratique, $\mathrm{T}_{2}$ constituerait la dose optimale de déjections de lapin à recommander pour une bonne production de macroinvertébrés benthiques d'eau douce en milieu contrôlé puisqu'il donne la meilleure production de macroinvertébrés, tout en offrant des conditions optimales de développement.

\section{Remerciements}

Nous exprimons notre gratitude au Ministère de l'Enseignement Supérieur et de la Recherche Scientifique du Bénin qui a octroyé une bourse à Richard Adande dans le projet « Appui aux doctorants ».

\section{Bibliographie}

Adande R.,Adjahouinou D.C.,Liady M.N.D.\& Fiogbe E.D., 2017. Alimentation des lapins (Oryctolagus cuniculus L.) à base de Azolla filiculö̈des, Elaeis guineensis, Ipomoea aquatica et Panicum maximum : effet sur la croissance des lapins et potentiel nutritif des crottes pour l'aquaculture. Int. J. Biol. Chem. Sci., 11, 2914-2923.

Agadjihouèdé H., Montchowui E., Chikou A. \& Laleye P.A., 2011. Libération comparée de sels dans l'eau par la minéralisation de l'azolla, la bouse de vache, la fiente de volaille et les sons de riz et de maïs utilisés en pisciculture. Int. J. Biol. Chem. Sci., 5(5), 1883-1897.

Akodogbo H.H., Bonou C.A. \& Fiogbe E.D., 2016. Production of freshwater benthic macroinvertebrates from pig dung: fertilization effect and optimal dose research. Int. J. Biol. Chem. Sci., 10(1), 242-254.
Angelier E., 2003. Ecology of streams and rivers. Enfield, CT, USA: Science Publishers Inc.

Benke A.C., Huryn A.D., Smock L.A. \& Wallace J.B., 1999. Length-mass relationships for freshwater macroinvertebrates in North America with particular reference to the Southeastern United States. J. North Am. Benthological Soc., 18(3), 308-343.

Biswas J.K., Ranaa S., Bhakta J.N. \& Jana B.B., 2009. Bioturbation potential of chironomid larvae for the sediment-water phosphorus exchange in simulated pond systems of varied nutrient enrichment. Ecol. Eng., 35, 1444-1453.

Bogut I. et al., 2007. Chironomus plumosus larvae, a suitable nutrient for freshwater farmed fish. Poljoprivreda, 13(1), 159-162.

Cartier V., Claret C., Garnier R. \& Franquet E., 2011. How salinity affects life cycle of a brackish water species Chironomidae salinarius (Diptera: Chironomidae). J. Exp. Mar. Biol. Ecol., 405(1-2), 93-98.

Cunha-Santino M.B.\& Bianchini Jr.I., 2003. Effect of initial concentration of dissolved oxygen in aeration coefficient for long-term BOD experiments. Acta Scientiarum Biol. Sci.Mar., 25(2), 253-256.

de Meutter F.V., Stoks R. \& De Meester L., 2005. The effect of turbidity state and microhabitat on macroinvertebrate assemblages: a pilot study of six shallow lakes. Hydrobiologia, 542, 379-390.

Epele L.B., Miserendino M.L. \& Band C., 2012. Does nature and persistence of substrate at a mesohabitat scale matter for Chironomidae assemblages? a study of two perennial mountain streams in Patagonia, Argentina. J. Insect. Sci., 12, article 68.

Evangelista A.D., Fortes N.R. \& Santiago C.B., 2005. Comparison of some live organisms and artificial diet as feed for Asian catfish Clarias macrocephalus (Günther) larvae. J. Appl. Ichthyol., 21, 437-443.

Fierro P. et al., 2017. Chapter 2: macroinvertebrates and fishes as bioindicators of stream water pollution. In: Hlanganani T., ed. Water quality. London: IntechOpen Limited, 23-38.

Hoómann A., 2005. Dynamic of Fine Particulate Organic Matter (FPOM) and macroinvertebrates in naturel artificial leaf packs. Hydrobiologia, 549, 167-178.

Hussain Q.A. \& Pandit A.K., 2012. Macroinvertebrates in streams: a review of some ecological factors. Int. J. Fish. Aquacult., 4(7), 114-123.

Kelly H.A.W. et al., 2013. Macroinvertebrate diets reflect tributary inputs and turbidity-driven changes in food availability in the Colorado River downstream of Glen Canyon Dam. Freshwater Sci., 32(2), 397- 410.

Kuhlmann M.L., Brandimarte A.L., Shimizu G.Y. \& Anaya M., 2001. Invertebrados bentônicos como indicadores de impactos antropicos sobre ecossistemas aquàticos continentais. In: Bollman H.A. et al. Indicadores ambientais: conceitos e aplicaçòes. Saõ Paulo, Brazil: EDUC/COMPED/INEP, 237-248. 
Leite-Rossi L.A. \& Trivinho-Strixino S., 2012. Are sugarcane leaf-detritus well colonized by aquatic macroinvertebrates? Acta Limnologica Brasiliensia, 24(3), 303-313.

Leuven R.S.E.W.,Brock T.C.M.\& Van Druten H.A.M., 1985. Effects of preservation on dry and ash-free dry weight biomass of some common aquatic macroinvertebrates. Hydrobiologia, 127, 151-159.

Liady M.N.D. et al., 2015. About the interest of a zooplankton compartment in pond systems: methodology to study the growth of D.pulex. Water Sci. Technol., 71(10), 14361443.

López van Oosterom M.V. et al., 2013. Trophic relationships between macroinvertebrates and fish in a pampean lowland stream (Argentina). Iheringia Sér. Zool., 103(1), 57-65.

Maleknejad R., Sudagar M. \& Azimi A., 2014. Étude comparative de l'effet de différents régimes d'alimentation sur la biomasse des larves de chironomides et la composition biochimique. Int. J. Adv. Biol. Biom. Res., 2(4), 1274-1278.

Matěna J., Šímová I., Brom J. \& Novotná K., 2016. Benthic communities in inland salinized waters with different salinities and nutrient concentrations and the ecology of Chironomus aprilinus (Diptera: Chironomidae) in the Czech Republic. Eur. J. Entomol., 113, 122-129.

Miserendino M.L., 2001. Length mass relationships for macroinvertebrates in freshwater environments of Patagonia (Argentina). Ecol. Austral, 11, 3-8.

Nath S., 2015. Relation between water quality and protein content of Chironomidae larvae. Proc. Zool. Soc., 68(2), 227-230.

Passel H.D., Dahm C.N. \& Bedrick E.J., 2007. Ammonia modelling for assessing potential toxicity to fish species in the Rio Grande. Ecol. Appl., 17, 2087-2099.

Ramesh C.S. \& Jitendra S.R., 2009. Monitoring of aquatic macroinvertebrates as bioindicator for assessing the health of wetlands: a case study in the Central Himalayas, India. Ecol. Indic., 9, 118-128.

Rey-Valette H., 2014. Quelques pistes sur l'avenir de l'aquaculture française en 2040. Cah. Agric., 23, 34-46.

Risnoveanu G., Postolache C. \& Vadineanu A., 2004. Ecological significance of nitrogen cycling by tubificid communities in shallow eutrophic lakes of the Danube Delta. Hydrobiologia, 524, 193-202.

Rodier J.B.L. \& Merlet N. \& coll., 2012. Analyse de l'eau. $9^{e}$ éd. Paris : Dunod.

Rosa B.J.F.V., Rodrigues L.F.T., de Oliveira G.S. \& da Gama Alves R., 2014. Chironomidae and Oligochaeta for water quality evaluation in an urban river in southeastern Brazil. Environ. Monit. Assess., 186(11), 7771-7779.

Rurangwa E., Van den Berg J. \& Laleye Ph., 2014. Mission exploratoire: pêche, pisciculture et aquaculture au Bénin, un quick scan du secteur pour des possibilités d'interventions. IMARES report C072/14 LEI report 14049, http://edepot.wur.nl/300319, (12/09/2018).
Sanseverino M.A. \& Nessimian J.L., 2008. The food of larval Chironomidae (Insecta, Diptera) in submerged litter in a forest stream of the Atlantic Forest (Rio de Janeiro, Brazil). Acta Limnol. Bras., 20(1), 15-20.

Schlumberger O. \& Bouretz N., 2002. Réseaux trophiques et production piscicole en étangs fertilisés (Dordogne, France). Rev. Sci.Eau, 1(15), 177-192.

Serra S.R.Q. et al., 2016. Synthesising the trait information of European Chironomidae (Insecta: Diptera): towards a new database. Ecol. Indic., 61, 282-292.

Shaw P.C. \& Mark K.K., 1980. Chironomid faming-a means of recycling farm manure and potentially reducing water pollution in Hong Kong. Aquaculture, 21, 155-163.

Shortle J.S., Abler D.G. \& Ribaudo M.A.R.K., 2001. 1. Agriculture and water quality: the issues. In: Shortle J.S. \& Abler D.G., eds. Environmental policies for agricultural pollution control. Cabi Publishing, 1-18.

Sorgeloos P., 2014. L'aquaculture a-t-elle le potentiel pour devenir la «biotechnologie bleue» dans l'avenir ? Cah. Agric., 23, 53-64.

Struijs J. et al., 2010. Field sensitivity distribution of macroinvertebrates for phosphorus in inland waters integrated. Environ. Assess. Manage., 7(2), 280-286.

Sulistiyarto B. \& Yulintine I.C., 2014. Developing production technique of bloodworm (Chironomidae larvae) in floodplain waters of fish feed. Int. J. Fish. Aquacult., 6(4), 39-45.

Thomaz S.M. et al., 2008. Influence of aquatic macrophyte habitat complexity on invertebrate abundance and richness in tropical lagoons. Freshwater Biol., 53, 358-367.

Tokeshi M., 1995. Production ecology. In: Cranston P.D., Armitage P.S. \& Pinder L.C.V., eds. The Chironomidae: biology and ecology of non-biting midges. Madras, India: Chapman \& Hall India, 269-296.

Uherek C.B. \& Gouveia F.B.P., 2014. Biological monitoring using macroinvertebrates as bioindicators of water quality of Maroaga stream in the Maroaga cave system, Presidente Figueiredo, Amazon, Brazil. Int. J. Ecol., 2014, ID 308149.

UNDESA (United Nations, Department of Economic and Social Affairs, Population Division), 2013. World population prospects: the 2012 revision, highlights and advance tables. New York, NY, USA: United Nations.

Waite I.R. et al., 2010. Comparison of watershed disturbance predictive models for stream benthic macroinvertebrates for three distinct ecoregions in western US. Ecol. Indic., 10, 1125-1136.

Waldbauer G.P., 1968. The consumption and utilization of food by insects. Adv. Insect Physiol., 5, 229-282.

Wang N. et al., 2007. Chronic toxicity of copper and ammonia to juvenile freshwater mussels (Unionidae). Environ. Toxicol. Chem., 26, 2048-2056.

Weiner E.R., 2012. Applications of environmental aquatic chemistry : a pratical guide. $3^{\text {rd }}$ ed. Boca Raton, FL, USA: CRC Press.

(48 réf.) 